\title{
Advances of Sensitive Infrared Detectors with HgTe Colloidal Quantum Dots
}

\author{
Shuo Zhang, Yao Hu and Qun Hao * \\ Beijing Key Laboratory for Precision Optoelectronic Measurement Instrument and Technology, \\ School of Optics and Photonics, Beijing Institute of Technology, Beijing 100081, China; \\ 3120195342@bit.edu.cn (S.Z.); huy08@bit.edu.cn (Y.H.) \\ * Correspondence: qhao@bit.edu.cn
}

Received: 8 July 2020; Accepted: 30 July 2020; Published: 4 August 2020

\begin{abstract}
The application of infrared detectors based on epitaxially grown semiconductors such as $\mathrm{HgCdTe}$, InSb and InGaAs is limited by their high cost and difficulty in raising operating temperature. The development of infrared detectors depends on cheaper materials with high carrier mobility, tunable spectral response and compatibility with large-scale semiconductor processes. In recent years, the appearance of mercury telluride colloidal quantum dots (HgTe CQDs) provided a new choice for infrared detection and had attracted wide attention due to their excellent optical properties, solubility processability, mechanical flexibility and size-tunable absorption features. In this review, we summarized the recent progress of HgTe CQDs based infrared detectors, including synthesis, device physics, photodetection mechanism, multi-spectral imaging and focal plane array (FPA).
\end{abstract}

Keywords: HgTe Colloidal quantum dots; infrared; photoconductors; photovoltaic; multispectral imaging

\section{Introduction}

Infrared detectors are widely used in thermal imaging [1,2], material spectroscopy analysis [3-5], autonomous driving assistants [6], surveillance [7] and biological health monitoring [8-10]. Before 1940s, the dominant infrared detectors were thermal detector, such as thermocouple detector [11], bolometer [12] and pyroelectric detector [13], which mainly relied on the detection of incident power rather than the energy of the incoming photons. Despite that they can be operated at room temperature, thermal detectors suffer from slower response speed and lower detectivity compared with photon detectors. Driven by the demands for fast response and high sensitivity, photon detectors gradually occupied the dominant position of infrared detection technology. The first photon detector was a PbS detector with a response wavelength of $3 \mu \mathrm{m}$, and germanium-mercury alloy (Ge:Hg), lead selenide (PbSe), germanium-silicon alloy (Ge:Si), indium antimonide ( $\mathrm{InSb}$ ) and mercury cadmium telluride $(\mathrm{HgCgTe})$ infrared detectors were successively developed. Infrared detectors and imaging systems have gone through "scan to image" and "single-color focal plane array (FPA)", and currently are moving towards large-format, multi-color 3rd and 4th generations [14]. Figure 1 showed the development roadmap of infrared detectors. The first generation of infrared detectors systems were relatively small refrigeration type photodetector unit or linear array, used with a complex two-dimensional scanning system to obtain images $[15,16]$. Compared with the first generation systems, the second generation systems had more pixels distributed on the two-dimensional FPA [17]. These pixels and readout integrated circuits (ROICs) constituted the infrared detection system, imaging by staring system electrically scanned. The third and fourth generation systems are being developed and are not well defined. In general, these systems are required to have large number of pixels, high frame rate, multi-color detection, integrated signal processing and other powerful functions. 


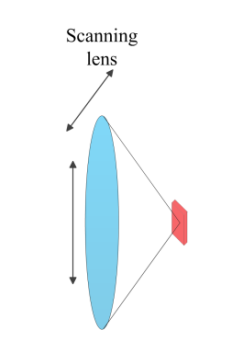

$1^{\text {st }}$ Gen

Scan to image

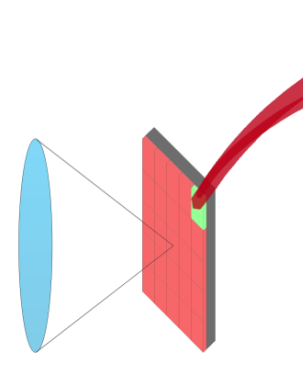

$2^{\text {nd }} \mathrm{Gen}$

FPA+ROIC

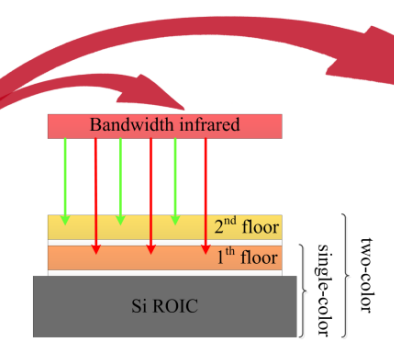

$3^{\text {rd }} \mathrm{Gen}$

FPA+ROIC

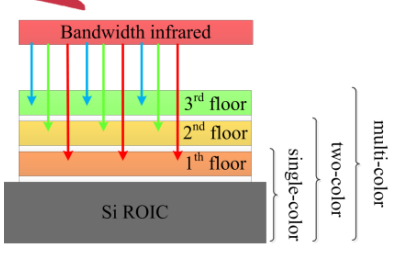

$4^{\text {th }}$ Gen

FPA+ROIC

Figure 1. The development roadmap of infrared detectors. Through scan to image and single-color FPA towards two-color and multi-color.

Nowadays, thermal imaging relies primarily on bulk epitaxial semiconductor materials. In mid-wave infrared (MWIR, 3-5 $\mu \mathrm{m}$ ) and long-wave infrared (LWIR, 8-12 $\mu \mathrm{m}$ ), it is dominated by narrow bandgap semiconductor material such as InSb [18], HgCgTe [19,20], quantum-wells [21] and type-II superlattices [22,23]. However, these materials were typically prepared by molecular beam epitaxy or chemical vapor deposition, which leads to high fabrication and processing costs. The applications of epitaxial materials-based infrared detectors are, therefore, limited to defense and scientific research, and there is an urgent need for new infrared materials that combines fast response, high sensitivity and compatibility with CMOS semiconductor processes to promote civilian applications, such as driverless car, atmospheric monitoring, thermal imaging, agricultural planting, etc.

As an alternative to conventional bulk epitaxial semiconductors, colloidal quantum dots (CQDs) have attracted wide attention due to their unique advantageous features such as the ease of solution-processing, low-cost and large-scale synthesis and size-tunable optical absorption wavelength. Over the past decade, CQDs have been widely used in a variety of applications, including solar cells [24], spectrometers [25], phototransistors [26], FPA imagers [27], lasers [28], LEDs [29]. Among all the CQDs systems, mercury telluride ( $\mathrm{HgTe}$ ) CQDs have demonstrated the highest infrared spectral absorption tunability covering main important atmospheric windows including short-wave infrared (SWIR, 1.5-2.5 $\mu \mathrm{m}$ ) [30-33], mid-wave infrared (MWIR, 3-5 $\mu \mathrm{m}$ ) [34-42], long-wave infrared (LWIR, $8-12 \mu \mathrm{m})[43,44]$ and even the terahertz $(\mathrm{THz})[45,46]$, appearing as promising candidates to substitute conventional semiconductors to achieve good detection performance with low cost. This review will be described from the following aspects, including: synthesis of infrared CQDs, infrared CQDs photodetectors, multispectral CQDs photodetectors and CQDs FPA.

\section{Synthesis of Infrared CQDs}

HgTe CQDs, a kind of zero-gap intrinsic semiconductor in the bulk, had a dominant position in the mid-infrared band of colloidal nanomaterials with interband optical transitions. In principle, the band gap can be adjusted in the full infrared range by controlling CQDs size. The synthesis [34,35] and optical properties [47] of HgTe CQDs with band-gaps tunable through the mid-infrared range of 3-5 $\mu \mathrm{m}(0.2$ to $0.5 \mathrm{eV})$ were first reported by Guyot-Sionnest group in the University of Chicago. The typical synthesis of monodispersed HgTe CQD involved the reaction of telluride (Te) and mercury chloride $\left(\mathrm{HgCl}_{2}\right)$ in non-polar solvents [35]. In brief, Te was dissolved in trioctylphosphine (TOP) and $\mathrm{HgCl}_{2}$ was dissolved in oleylamine, forming a clear, colorless or pale-yellow solution. To initiate the reaction, TOP:Te was rapidly injected in to $\mathrm{HgCl}_{2}$ solution. After a period of reaction time, the reaction was quenched by adding dodecanethiol and the final particle size depended on the temperature and duration of the reaction, typically in the range of 60 to $130{ }^{\circ} \mathrm{C}$ and 1 to $40 \mathrm{~min}$. Transmission electron microscopy (TEM) image of HgTe CQDs with shape of triangle or distorted parallelogram is shown in Figure 2a. Absorption and photodetection with well-defined edges, as well as narrow photoluminescence, were tunable across the near and mid-IR between 1.3 and $5 \mu \mathrm{m}$. The typical infrared absorption spectra of different sizes are shown in Figure $2 b$. 

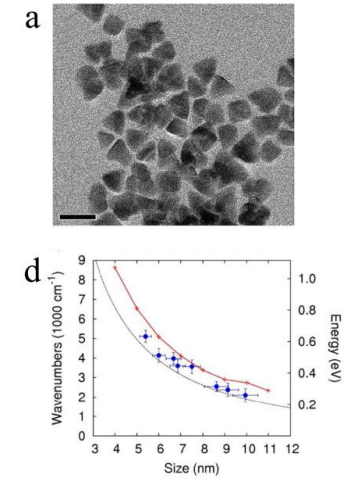
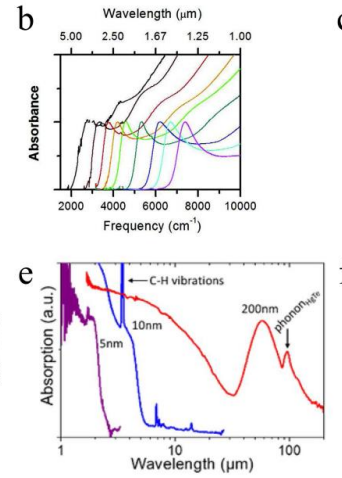
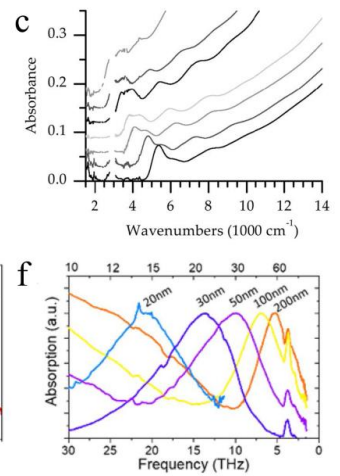

Figure 2. (a) Transmission electron microscopy image of HgTe CQOs, scale bar is $20 \mathrm{~nm}$, adapted with permission from [35]; Copyright 2011 American Chemical Society. (b) The spectral absorption of HgTe CQDs with cut-off wavelength up to $5 \mu \mathrm{m}$, adapted with permission from [35]; Copyright 2011 American Chemical Society. (c) Absorption spectra of particles of different sizes show better spectral resolution, adapted with permission from [43]; Copyright 2014 American Chemical Society. (d) Blue dots: A functional relationship between the photoluminescence peak energy and the CQDs size measured in TEM images. Red line and crosses: calculation of exciton gap of truncated tetrahedral shape CQDs. Black dotted line: calculation of exciton gap versus diameter of spherical CQDs. Adapted with permission from [43]; Copyright 2014 American Chemical Society. (e) Absorption spectra of various sizes HgTe CQD, adapted with permission from [45]; Copyright 2018 American Chemical Society. (f) Absorption spectra of various sizes HgTe CQDs, presenting an intraband/plasmonic characteristic in the LWIR, very long-wavelength infrared range (VLWIR, 12-20 $\mu \mathrm{m}$ range) or THz range, adapted with permission from [45]; Copyright 2018 American Chemical Society.

In 2014, the Guyot-Sionnest group further improved the synthesis method of HgTe CQDs by making a slight modification by TOP:Te precursor dilution with oleylamine, which enabled it to display better spectral resolution, as shown in Figure 2c [43]. A more homogeneous mixture is formed by diluting Te precursors before appreciable growth occurs, and additional nucleation is prevented by sufficient cooling [43]. Through regrowing of particles by dropwise addition of $\mathrm{HgCl}_{2}$ and TOP:Te dissolved in oleylamine at $110-120^{\circ} \mathrm{C}$, the absorption of LWIR was realized. The HgTe CQDs size dependence of the energy gaps was shown in Figure 2d. In 2017, a new method for synthesizing $\mathrm{HgTe}$ CQDs using more active tellurium sources and excess mercury precursors was reported by Guyot-Sionnest group, to produce more spherical and non-agglomerating tendency HgTe CQDs, which showed air-stable n-doping [48]. In 2018, Lhuillier Group in Sorbonne Universite reported a synthesis method of HgTe CQD with an absorption cutoff wavelength covering the whole infrared range from $2 \mu \mathrm{m}$ up to the THz range $(65 \mu \mathrm{m})$, with corresponding $\mathrm{HgTe}$ CQD sizes ranging from $5 \mathrm{~nm}$ to above $200 \mathrm{~nm}$ [45], as shown in Figure 2e,f.

\section{Infrared CQDs Photodetectors}

CQDs have emerged as a powerful and versatile platform for building electronic and optoelectronic devices with their excellent tunable optoelectronic properties and low cost and large-scale synthesis. HgTe CQDs infrared photodetectors mainly include three categories: photoconductors, phototransistors and photovoltaics. The first reported HgTe CQDs photodetector is a photoconductor [34]. When the photon energy is greater than the band gap, the conductivity of the CQDs can be controlled by absorbing photons. The controls of surface ligand are a key step toward the design of photoconductive thin films. In most cases, CQDs synthesis requires the use of long-chain organic ligands, which can ensure the stable growth of the nanometer size of CQDs and enable the CQDs to overcome the van der Waals interparticle attraction for better stabilization in the form of colloids. However, long-chain ligands are not suitable for electronic applications. Initial long-chain ligands need to be exchanged 
by short-chain ligands to increase the inter-CQD coupling and obtain reasonable carrier mobility to increase the conductivity.

A more powerful infrared detector should be able to give a higher specific detectivity $D^{*}$. A high $D^{*}$ means that the detector has a strong ability to detect weak signals. The $D^{*}$ can be calculated as

$$
D^{*}=\frac{(A \Delta f)^{1 / 2}}{N E P}=\frac{(A \Delta f)^{1 / 2}}{i_{n}} R
$$

where $A$ is detector area, $\Delta f$ is the amplifier bandwidth, NEP is the noise equivalent power, $i_{\mathrm{n}}$ is the noise current and $R$ is the responsivity. The responsivity is the ratio of the output voltage or current of the detector to the radiated power incident on the photosensitive region of the detector, can be calculated as:

$$
\begin{aligned}
& R=\frac{I_{\text {out }}}{P} \\
& R=\frac{V_{\text {out }}}{P}
\end{aligned}
$$

where $I_{\text {out }}$ is the output current of the detector, $V_{\text {out }}$ is the output current voltage of the detector and $P$ is the radiated power

The device architecture of the photoconductor is the simplest, which can be fabricated by depositing CQDs on a prefabricated interdigitated electrode [49], as shown in Figure 3a. Although the structure of the photoconductive device is simple, the performance of the device suffered from dark-current and $1 / f$ noise [50], which limited the specific detectivity $\left(D^{*}\right)$ of the mid-infrared HgTe CQDs photoconductors on the order of a few $10^{9}$ Jones at $80 \mathrm{~K}$ [34].

The performance of CQDs photoconductors can be improved by using compact ligands, which allows higher mobility state. The hybrid ligand exchange and polar phase transfer technology were used to realize the improved mid-infrared photoconductors based on HgTe CQDs, which improved the mobility of carriers and accurately tuned the doping level of HgTe CQDs, so that the $D^{*}$ of detector was increased to $4.5 \times 10^{10}$ Jones at $80 \mathrm{~K}$ and $500 \mathrm{~Hz}$, and a $5 \mu \mathrm{m}$ cutoff wavelength [42].

Similar to photoconductors, the amount of photocurrent in a phototransistor varies according to the intensity of light, whereas phototransistors combined a biased high-mobility channel such as graphene and $\mathrm{MoS}_{2}$ to achieve photoconductivity gain. The photoconductivity gain represents the ratio of the external photocurrent formed by the photo-generated carriers under bias voltage to the internal photocurrent formed by the photoelectron. Hybrid device architectures such as grapheme-CQDs [26,51], $\mathrm{MoS}_{2}$-CQDs [52,53] and grapheme- $\mathrm{MoS}_{2}$ [54] of phototransistors have been reported, demonstrating high photoconductivity gains and ultra-high optical sensitivities. However, the photoconductive gain amplifies not only the photocurrent but also any noise due to thermal excitation or recombination. The structure schematic diagram of a $\mathrm{MoS}_{2}$-CQDs hybrid device is shown as Figure 3b.

Compared with HgTe CQDs photoconductors, the photovoltaic devices improved device speed and sensitivity, and theoretically avoided 1/f noise and dark current. In 2015, Guyot-Sionnest group developed the first background-limited infrared photodetection (BLIP) HgTe CQD mid-wave infrared detector, which achieved $D^{*}$ of $4.2 \times 10^{10}$ Jones, microseconds response times and covered all the infrared spectrum regions with a cut-off at $5.25 \mu \mathrm{m}$ at $90 \mathrm{~K}$ [38]. Figure $3 \mathrm{c}$ give an illustration of the first BLIP HgTe CQDs photovoltaic detector and the function of current of the detector with the applied bias, at different ambient temperatures. In 2018, Guyot-Sionnest group further improved the sensitivity, efficiency, and response speed of the photovoltaic MWIR detectors through a new doping method [40]. A solid-state cation exchange method was introduced to achieve heavily p-doped HgTe CQDs by using $\mathrm{Ag}_{2} \mathrm{Te}$ nanocrystals. The specific doping method is to spin-coating $\mathrm{Ag}_{2} \mathrm{Te}$ nanocrystals onto $\mathrm{HgTe}$ CQDs and expose them to $\mathrm{HgCl}_{2}$. $\mathrm{Ag}_{2}$ Te nanocrystals liberate $\mathrm{Ag}^{+}$ions and then form $\mathrm{AgCl}_{2}$ and immobilize them on the surface of HgTe CQDs. This doping scheme increases the $\mathrm{D}^{*}$ of the device by one or two orders of magnitude [40]. Schematic and cross-sectional Scanning electron microscopy image of the HgTe CQDs photodetectors are shown in Figure 3d,e [40]. The MWIR absorption in the 
typical thickness of $\sim 400 \mathrm{~nm}$ HgTe film in the device is $\sim 18 \%$ and the corresponding internal quantum efficiency (IQE) was estimated to be as high as 90\% [40]. To further improve device performance, some optical structures have been reported, such as optical nano-antennas [39], plasmonic nano-disk array [41] and gold nanorods [55], which increased the $D^{*}$ by two or three times. Figure $3 f$ showed the schematic of the HgTe CQDs midwave infrared detector with enhanced light absorption by plasmonic nano-disks [41]. Mechanical flexibility and easy integration with flexible substrates without lattice matching is another advantage of CQDs over traditional semiconductor materials. In 2019, Tang et al. fabricated the first flexible HgTe CQDs photovoltaic detector on a polyimide substrate and integrated a Fabry-Perot resonator cavity to further improved detectivity [56]. The Fabry-Perot resonant cavity is composed of the reflection layer at the bottom, the gold semi-reflector at the top and the CQDs, ITO and optical spacer sandwiched in the middle [56]. The infrared light illuminated from the top bounced back and forth between the reflective layer at the bottom and the semi-reflective layer at the top, enhanced the infrared light at specific wavelength. The schematic of the flexible device is shown in Figure 3g. Besides enhancing absorption, optical structures can also be used for better spectral resolution. By combining HgTe CQD photovoltaic sensors with distributed Bragg mirror filter array in a single device, Tang et al, reported a CQDs hyperspectral sensor [57], which realized acquisition of hyperspectral data with 64 narrowband channels and full-width at half-maxima down to $30 \mathrm{~cm}^{-1}$, as shown in Figure 3h.

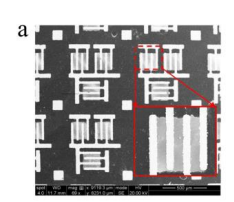

d

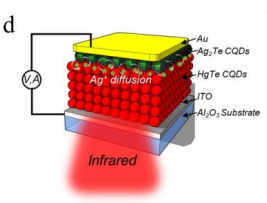

g

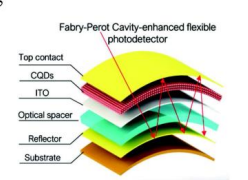

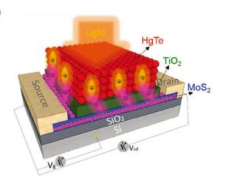

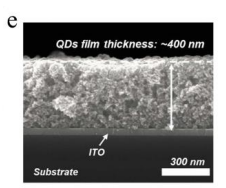

h

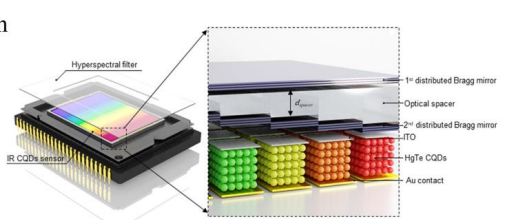

Figure 3. (a) Scanning electron microscopy image of a photoconductor by depositing CQDs on a prefabricated interdigitated electrode, adapted with permission from [49]; Copyright 2016 American Chemical Society. (b) Schematic of the fast and sensitive HgTe CQDs photodetectors: The structure schematic diagram of a MoS 2 -CQDS hybrid device, adapted with permission from [52]; Copyright 2017 WILEY-VCH Verlag GmbH \& Co. KGaA, Weinheim. (c) Illustration of the first BLIP HgTe CQDs photovoltaic detector. The function of current of the photoelectric device with the applied bias, at $90 \mathrm{~K}$ in the dark (black line), receiving environment $295 \mathrm{~K}$ radiation (blue line) and receiving $873 \mathrm{~K}$ blackbody lighting (red line). Reprinted with permission from [38]; Copyright 2015 AIP Publishing LLC. (d) Schematic of the fast and sensitive HgTe CQDs photodetectors, adapted with permission from [40]; Copyright 2018 American Chemical Society. (e) Cross-sectional Scanning electron microscopy image of the fast and sensitive HgTe CQDs photodetectors, adapted with permission from [40]; Copyright 2018 American Chemical Society. (f) Schematic of HgTe CQDs mid-wave infrared detector with enhanced light absorption by plasmonic disks, adapted with permission from [41]; Copyright 2018 American Chemical Society. (g) Schematic of a flexible HgTe CQDs photodetector with enhanced light absorption through a Fabry-Perot cavity, adapted with permission from [56]; Copyright 2019 WILEY-VCH Verlag GmbH \& Co. KGaA, Weinheim. (h) CQDs hyperspectral detector was composed of HgTe CQDs detector and hyperspectral filter, adapted with permission from [57]; Copyright 2019 WILEY-VCH Verlag GmbH \& Co. KGaA, Weinheim. 
In order to better reflect the development of infrared CQDs photodetectors, Table 1 shows the performance comparison between HgTe CQDs photodetectors over the past decade.

Table 1. Performance comparison between HgTe CQDs photodetectors.

\begin{tabular}{|c|c|c|c|c|c|c|c|}
\hline Device Structure Type & Year & Spectral Range $(\mu \mathrm{m})$ & $R(\mathrm{~A} / \mathrm{W})$ & EQE (\%) & $D^{*}$ (Jones) & Response Time & Reference \\
\hline HgTe CQDs photoconductors & 2011 & $<5$ & 0.25 & 10 & $2 \times 10^{9}$ & NA & [34] \\
\hline HgTe CQDs photoconductors & 2014 & $<12$ & $3 \times 10^{-4}$ & NA & $6.5 \times 10^{6}$ & $<5 \mu \mathrm{s}$ & [43] \\
\hline HgTe CQDs photoconductors & 2019 & $<5$ & 0.2 & 30 & $4.5 \times 10^{10}$ & NA & [42] \\
\hline $\mathrm{HgTe} / \mathrm{AsS}_{3}$ phototransistors & 2013 & $<3.5$ & $5 \times 10^{-3}$ & NA & $3.5 \times 10^{10}$ & NA & [37] \\
\hline HgTe CQDs phototransistors & 2017 & $<2$ & 0.4 & NA & $2 \times 10^{10}$ & NA & [31] \\
\hline $\mathrm{MoS}_{2}-\mathrm{HgTe}$ CQDs Hybrid phototransistors & 2017 & $<2$ & $10^{6}$ & NA & $10^{12}$ & $4 \mathrm{~ms}$ & [52] \\
\hline HgTe CQDs photodiodes & 2015 & $3-5$ & $8 \times 10^{-2}$ & 2.5 & $4.2 \times 10^{10}$ & $0.7 \mu \mathrm{s}$ & [38] \\
\hline HgTe CQDs photodiodes & 2018 & $<4.8$ & 0.38 & 17 & $1.2 \times 10^{11}$ & $<1 \mu \mathrm{s}$ & [40] \\
\hline $\begin{array}{l}\text { Plasmon ResonanceEnhanced HgTe } \\
\text { CQDs photodiodes }\end{array}$ & 2018 & $<4.5$ & 1.62 & 45 & $4 \times 10^{11}$ & $<1 \mu \mathrm{s}$ & [41] \\
\hline Flexible HgTe CQDs photodiodes & 2019 & $<2.2$ & 0.5 & 30 & $7.5 \times 10^{10}$ & $260 \mathrm{~ns}$ & [56] \\
\hline Hyperspectral HgTe CODs photodiodes & 2019 & $1.53-2.08$ & 0.2 & 11 & $>10^{10}$ & $120 \mathrm{~ns}$ & [57] \\
\hline Dual-band $\mathrm{HgTe} C Q D$ s photodiodes & 2019 & $<2.5$ and $3-5$ & 0.3 and 0.15 & NA & $10^{11}$ and $3 \times 10^{10}$ & $<2.5 \mu \mathrm{s}$ & [58] \\
\hline HgTe CQDs photodiodes & 2020 & $2-3$ & 1 & 30 & $10^{11}$ & $\mathrm{NA}$ & [33] \\
\hline
\end{tabular}

\section{Multispectral CQDs Photodetectors}

According to the development history of infrared detectors and systems, the third and fourth generation of infrared imaging requires simultaneous detection of two or more wavelengths in photoresponse. Multispectral detection provides better target identification and allows accurate determination of the infrared signature of the target.

By vertically stacking one SWIR and one MWIR HgTe CQD photodiode into an n-p-n structure, Tang et al. fabricated the first CQDs two-terminal dual-band infrared detector in 2019 to switch the device's spectral response between short-wave and mid-wave infrared by controlling the bias polarity and magnitude [58]. Compared to a three-terminal configuration using a common ground contact [2], this two-terminal structure increased the optical fill factor. The architecture and cross-sectional Scanning electron microscopy image of the two-terminal dual-band device were shown in Figure $4 \mathrm{a}, \mathrm{b}$ with $\mathrm{Bi}_{2} \mathrm{Se}_{3}$ and $\mathrm{Ag}_{2} \mathrm{Te}$ as the $\mathrm{n}$ and $\mathrm{p}$ layers. Figure $4 \mathrm{c}$ showed the simplified energy diagram of the device at zero bias, indicating the n-p-n structure of the device. When the bias voltage of the detector gradually changes from -300 to $+500 \mathrm{mV}$, the spectral response of the device gradually switched from SWIR to MWIR, as shown in Figure 4d. The two-terminal dual-band detector can produce SWIR image and MWIR image of object respectively, and can also generate the combined two-color image. The obvious two-color image can provide more information than the single image. The SWIR, MWIR and two-color composite images of cold and hot water were shown in Figure 4e. In addition to imaging, the two-terminal dual-band detector can be used to determine the temperature of object by the SWIR/MWIR signal ratio, which is more reliable than single-band detection.

Although the two-terminal dual-band detector with architecture of vertically stacked two different-sized HgTe CQD photodiodes [58] has superior performance, it is limited to two-color functionality. It is difficult to achieve more spectral channels with vertical stacks, which requires more complex band engineering. Although the spatial resolution and the optical fill factor would be reduced, pixelating CQDs of different sizes in different areas of a chip would seem to be a better solution for multicolor detection. Therefore, the key to achieving the transition from monochromatic detectors to multicolor detectors with CQDs is to develop CQDs patterning methods to selectively deposit different size CQDs with well-defined geometry in different areas. Several CQDs patterning techniques have been demonstrated to optimize the resolution, throughput and fidelity, including lithographical lift-off [59,60], contact printing [61], transfer printing [49,62,63], inkjet printing [30,64,65], screen printing [66] and laser writing [67].

A simple and efficient Poly(methyl methacrylate) (PMMA)-assisted transfer technique was reported for the scalable fabrication CQD-based multicolor photodetector with multi-pixels in response to different spectral ranges of infrared by using patterned HgTe CQD films [49], as shown in Figure 5a,b. However, during CQDs patterning, photoresist residue, developer, heating, or plasma etching may change the properties of CQDs. 
a

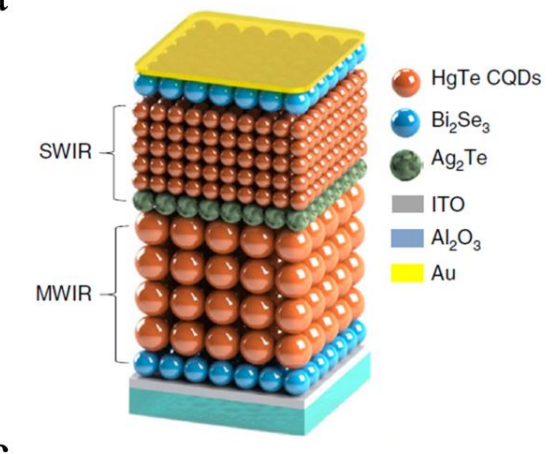

$\mathrm{c}$

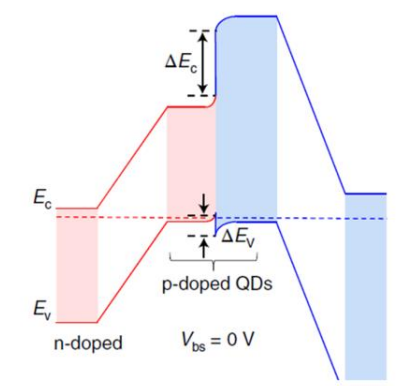

b
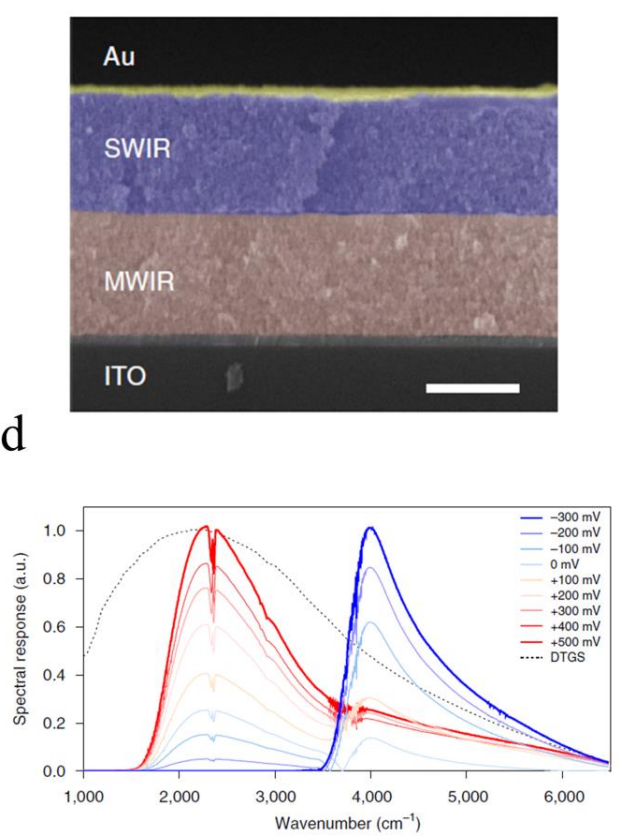

e

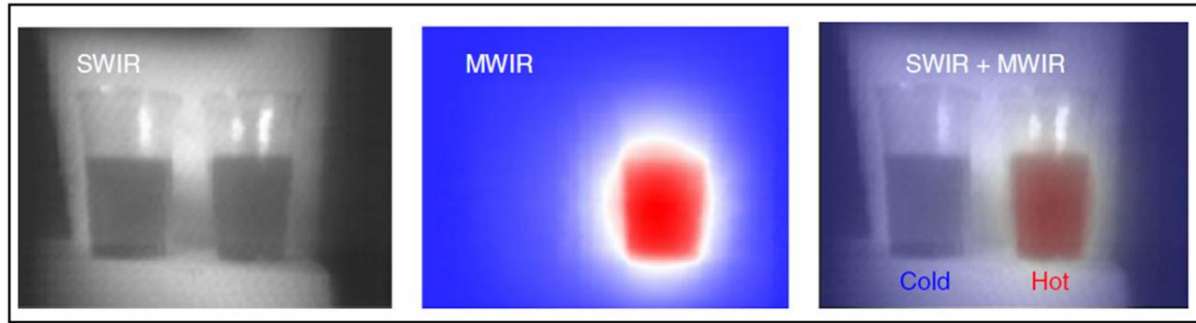

Figure 4. (a) Architecture of the two-terminal dual-band detector. (b) Cross-sectional Scanning electron microscopy image of the detector. Scale bar: $300 \mathrm{~nm}$. (c) Energy diagram of the two-terminal dual-band detector at zero bias. (d) The spectral response of the detector varies with bias voltage from -300 to $+500 \mathrm{mV}$. (e) Cold and hot water's SWIR, MWIR and two-color composite images. Adapted with permission from [58]; Copyright 2019 Nature Publishing Group.

Wang et al. reported a photoresists-free technique for direct optical patterning of CQDs, called direct optical lithography of functional inorganic nanomaterials (DOLFIN), and designed a series of photosensitive ligands to build an extensive library of functional photosensitive inorganic inks $[68,69]$. Under controlled light exposure, the compact photosensitive surface ligands of CQDs undergo chemical transformations that alter the solubility of CQDs in the developer. The resolution of the CQDs patterning produced by this method is comparable with that of traditional photolithography, without compromising the electronic and optical properties. Using similar patterning techniques, Kim et al. demonstrated a low-temperature fabricated two-dimensionally pixelized full-color photodetector by using monolithic integration of various-sized CQDs and amorphous indium-gallium-zinc-oxide semiconductors [70]. As a parallel technology, DOLFIN has various advantages, but specific ligands are required. Further verification is needed to determine whether the influence of DOLFIN on the synthesis of HgTe CODs and precise doping in HgTe CQDs photovoltaic devices, as well as whether DOLFIN can be applied to HgTe CQDs multi-spectral infrared detector. Looking at the various CQDs patterning techniques, inkjet printing appears to be the most promising for fabrication HgTe CQDs multi-spectral infrared detector. The key is to develop a fast and reliable parallel inkjet printing method. 
$\mathrm{a}$

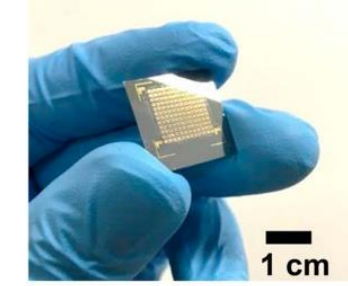

b

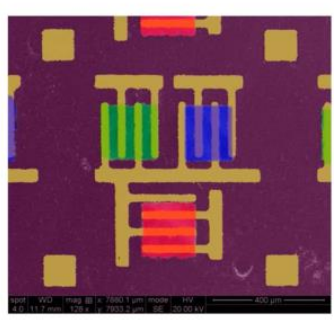

$\mathrm{c}$

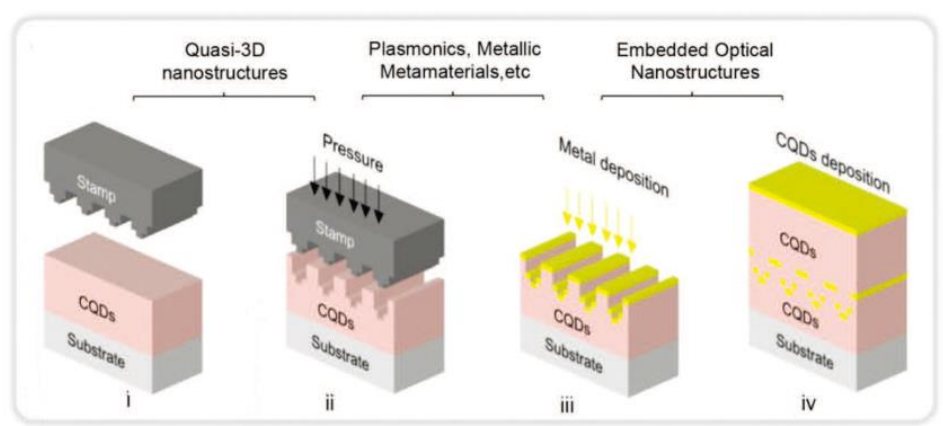

d
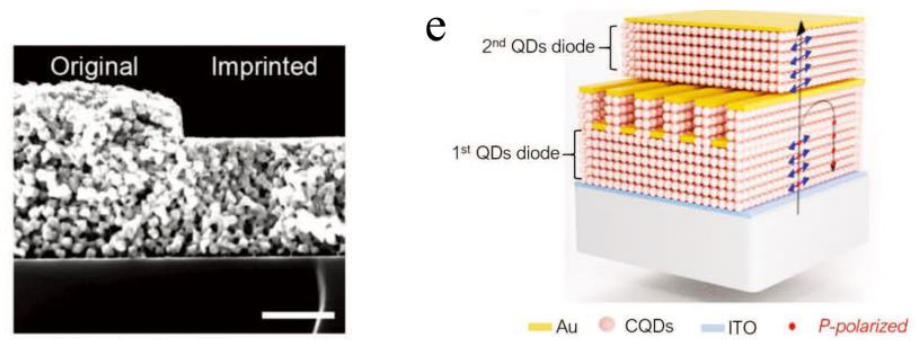

Figure 5. (a) A photo of a three-pixel photodetectors, adapted with permission from [49]; Copyright 2016 American Chemical Society. (b) False-colored Scanning electron microscopy image of the three-pixel photodetectors, adapted with permission from [49]; Copyright 2016 American Chemical Society. (c) Direct imprinting technique process, adapted with permission from [71]; Copyright 2020 WILEY-VCH Verlag GmbH \& Co. KGaA, Weinheim. (d) Cross-sectional scanning electron microscopy image of imprinted CQDs film. Scale bar is $100 \mathrm{~nm}$, adapted with permission from [71]; Copyright 2020 WILEY-VCH Verlag GmbH \& Co. KGaA, Weinheim. (e) Illustration of the two-terminal dual-channel infrared polarization detector with the imprinting technology, adapted with permission from [71]; Copyright 2020 WILEY-VCH Verlag GmbH \& Co. KGaA, Weinheim.

Besides 2D CQDs patterning techniques, Tang et al. reported a direct imprinting technique for producing high resolution CQDs quasi-3D optical nanostructures without degrading the key properties of materials [71]. The direct imprinting technique process was shown in Figure 5c. Firstly, nano-structured silicon stamps were prepared by using traditional semiconductor technology; the nanostructures were then transferred to the CQDs film by contacting the CQDs with the stamp and applying pressure; last, by metal deposition and capping another layer of CQDs, the high-resolution nanophotonic structure is embedded into the device. A cross-sectional scanning electron microscopy image of imprinted CQDs film is shown in Figure 5d. The imprinting pattern has clear and sharp edges, no large cracks or pile-ups of CQDs, and the surface roughness of the imprinting area is reduced, which is beneficial and necessary for high-precision patterning. With the imprinting technology, a two-terminal dual-channel infrared polarization detector was designed to detect both polarized and unpolarized light, as shown in Figure 5e. A bilayer wire-grid polarizer embedded in CQDs can be used as optical polarization filter and middle electrode for signal readout. 


\section{CQDs Focal Plane Array}

In conventional $\mathrm{HgCdTe}$ or InSb FPA infrared detectors, the active layer is prepared by high-temperature epitaxial growth, which is a very expensive technique, and must be coupled to ROIC by indium bumps, which further increases the cost. When building an FPA, the pixel size should be ideally just above the target wavelength to improve image quality. The pixel size of $\mathrm{HgCdTe}$ or InSb FPA infrared detectors is actually limited by technology and more specifically by the indium bump hybridization to the ROICs.

As an interesting candidate, semiconductor CQD can produce infrared detectors by simple drop-casting or spin-coating on a silicon ROICs, without molecular beam epitaxy and lattice-match with the substrate. This greatly reduces the cost of the monolithic FPA infrared detectors, which is an unmatched advantage over traditional semiconductors. In 2016, Guyot-Sionnest group reported the first CQDs FPA infrared detector for mid-infrared imaging by coupling the HgTe CQDs with a silicon ROICs [72]. A study by Guyot-Sionnest Group demonstrated a simple fabrication method for a low-cost HgTe CQDs FPA thermal camera with noise equivalent difference (NEDT) of $1.02 \mathrm{mK}$ at $5 \mu \mathrm{m}$ and achieved images at 120 frames per second using the CQDs FPA infrared detector [73]. CQDs have been demonstrated to be a promising material for infrared imaging due to its excellent optoelectronic properties, low cost, easy synthesis, and mechanical flexibility.

\section{Conclusions and Outlook}

After more than a decade of intensive research, HgTe CQDs can be considered as a possible alternative to conventional semiconductor infrared sensing. The main achievements have been demonstrated include full tunability of the absorption over the infrared range, the fast detectors with background-limited infrared photodetection, flexible and curved infrared detector with high $D^{*}$ and short response time, and the CQDs-based FPA in both SWIR and MWIR range.

CQDs bring both opportunities and challenges to the development of infrared detectors. Although synthetic improvements in CQDs are still possible, surface chemistry, film processing, and doping control require further research to achieve higher detection capabilities at higher temperatures. On the other hand, the CQDs infrared detectors reported at the present stage mainly focus on the SWIR and MWIR, while there are few reports on the LWIR and its performance is poor compared with that of commercial detectors. Therefore, the CQDs LWIR device is an urgent direction to be explored. At the same time, an efficient, accurate and large-scale CQDs patterning technology is needed to meet the requirements of FPA and multi-spectral imaging. For the future application of civilian and military infrared detectors, HgTe CQDs will meet many serious challenges. Most of the reported HgTe CQDs infrared detectors were single-pixel devices with less FPA and worse performance than commercial infrared detectors. For many systems such as night vision, wide range monitoring and astronomical applications that require large FPA and high pixel densities, an efficient CQDs pixelation method is required. In addition, the performance stability of HgTe CQDs infrared detector is a challenge in some extreme environments such as high temperature, high pressure and space environment.

Author Contributions: Investigation, S.Z.; writing—original draft preparation, S.Z.; writing—review and editing, Y.H. and Q.H.; project administration, Y.H.; funding acquisition, Q.H. All authors have read and agreed to the published version of the manuscript.

Funding: The work was supported by the National Natural Science Foundation of China (NSFC) (51735002 and 61875012).

Conflicts of Interest: The authors declare no conflict of interest.

\section{References}

1. Rogalski, A. Toward third generation HgCdTe infrared detectors. J. Alloy. Compd. 2004, 371, 53-57. [CrossRef]

2. Rogalski, A.; Antoszewski, J.; Faraone, L. Third-generation infrared photodetector arrays. J. Appl. Phys. 2009, 105, 091101. [CrossRef] 
3. Stouwdam, J.W.; Veggel, F.C.J.M.V. Near-infrared emission of redispersible $\mathrm{Er}^{3+}$, $\mathrm{Nd}^{3+}$, and $\mathrm{Ho}^{3+}$ doped $\mathrm{LaF}_{3}$ nanoparticles. Nano Lett. 2002, 2, 733-737. [CrossRef]

4. Schmitt, J.; Flemming, H.C. FTIR-spectroscopy in microbial and material analysis. Int. Biodeterior. Biodegrad. 1998, 41, 1-11. [CrossRef]

5. Madejová, J. FTIR techniques in clay mineral studies. Vib. Spectrosc. 2003, 31, 1-10. [CrossRef]

6. González, A.; Fang, Z.; Socarras, Y.; Serrat, J.; Vázquez, D.; Xu, J.; López, A.M. Pedestrian detection at day/night time with visible and FIR cameras: A comparison. Sensors 2016, 16, 820. [CrossRef]

7. Briz1, S.; de Castro, A.J.; Aranda, J.M.; Meléndez, J.; Lo' pez, F. Reduction of false alarm rate in automatic forest fire infrared surveillance systems. Remote Sens. Environ. 2003, 86, 19-29. [CrossRef]

8. Soref, R. Mid-infrared photonics in silicon and germanium. Nat. Photonics 2001, 4, 495-497. [CrossRef]

9. Miller, L.M.; Smity, G.D.; Carr, G.L. Synchrotron-based biological microspectroscopy: From the mid-infrared through the far-infrared regimes. J. Biol. Phys. 2003, 29, 219-230. [CrossRef]

10. Yokota, T.; Zalar, P.; Kaltenbrunner, M.; Jinno, H.; Matsuhisa, N.; Kitanosako, H.; Tachibana, Y.; Yukita, W.; Koizumi, M.; Someya, T. Ultraflexible organic photonic skin. Sci. Adv. 2016, 2, e1501856. [CrossRef]

11. Nelms, N.; Dowson, J. Goldblack coating for thermal infrared detectors. Sens. Actuators A 2005, 120, $403-407$. [CrossRef]

12. Langley, S.P. The Bolometer. Nature 1881, 25, 14-16.

13. Chynoweth, A.G. Dynamic method for measuring the pyroelectric effect with special reference to barium titanate. J. Appl. Phys. 1956, 27, 78. [CrossRef]

14. Martyniuk, P.; Antoszewski, J.; Martyniuk, M.; Faraone, L.; Rogalski, A. New concepts in infrared photodetector designs. Appl. Phys. Rev. 2014, 1, 41102. [CrossRef]

15. Elliott, C.T.; Day, D.; Wilson, D.J. An integrating detector for serial scan thermal imaging. Infrared Phys. 1982, 22, 31-42. [CrossRef]

16. Blackburn, A.; Blackman, M.V.; Charlton, D.E.; Dunn, W.A.E.; Jenner, M.D.; Oliver, K.J.; Wotherspoon, J.T.M. The practical realisation and performance of sprite detectors. Infrared Phys. 1982, 22, 57-64. [CrossRef]

17. Scribner, D.A.; Kruer, M.R.; Killiany, J.M. Infrared focal plane array technology. Proc. IEEE 1991, 79, 66-85. [CrossRef]

18. Camargo, E.G.; Ueno, K.; Morishita, T.; Sato, M.; Endo, H.; Kurihara, M.; Ishibashi, K.; Kuze, N. High-sensitivity temperature measurement with miniaturized InSb mid-IR sensor. IEEE Sens. J. 2007, 7, 1335-1339. [CrossRef]

19. Figgemeier, H.; Ames, C.; Beetz, J.; Breiter, R.; Eich, D.; Hanna, S.; Mahlein, K.M.; Schallenberg, T.; Sieck, A.; Wenisch, J. High-performance SWIR/MWIR and MWIR/MWIR bispectral MCT detectors by AIM. Infrared Technol. Appl. Xliv. 2018, 10624, 106240S. [CrossRef]

20. Cervera, C.; Baier, N.; Gravrand, O.; Mollard, L.; Lobre, C.; Destefanis, G.; Zanatta, J.P.; Boulade, O.; Moreau, V. Low-dark current p-on-n MCT detector in long and very long-wavelength infrared. Infrared Technol. Appl. Xli. 2015, 9451, 945129. [CrossRef]

21. Sarusi, G. QWIP or other alternative for third generation infrared systems. Infrared Phys. Technol. 2003, 44, 439-444. [CrossRef]

22. Haddadi, A.; Dehzangi, A.; Chevallier, R.; Adhikary, S.; Razeghi, M. Bias-selectable nBn dual-band long-/very long-wavelength infrared photodetectors based on InAs/InAs ${ }_{1-x} \mathrm{Sb}_{x} / \mathrm{AlAs}_{1-x} \mathrm{Sb}_{x}$ type-II superlattices. Sci. Rep. 2017, 7, 3379. [CrossRef]

23. Haddadi, A.; Chevallier, R.; Chen, G.; Hoang, A.M.; Razeghi, M. Bias-selectable dual-band mid-/long-wavelength infrared photodetectors based on InAs/InAs ${ }_{1-x} \mathrm{Sb}_{x}$ type-II superlattices. Appl. Phys. Lett. 2015, 106, 011104. [CrossRef]

24. Wang, X.; Koleilat, G.I.; Tang, J.; Liu, H.; Kramer, I.J.; Debnath, R.; Brzozowski, L.; Barkhouse, D.A.R.; Levina, L.; Hoogland, S.; et al. Tandem colloidal quantum dot solar cells employing a graded recombination layer. Nat. Photon. 2011, 5, 480-484. [CrossRef]

25. Bao, J.; Bawendi, M.G. A colloidal quantum dot spectrometer. Nature 2015, 523, 67-70. [CrossRef]

26. Konstantatos, G.; Badioli, M.; Gaudreau, L.; Osmond, J.; Bernechea, M.; de Arquer, F.P.G.; Gatti, F.; Koppens, F.H.L. Hybrid graphene-quantum dot phototransistors with ultrahigh gain. Nat. Nanotechnol. 2012, 7, 363-368. [CrossRef] 
27. Goossens, S.; Navickaite, G.; Monasterio, C.; Gupta, S.; Piqueras, J.J.; Pérez, R.; Burwell, G.; Nikitskiy, I.; Lasanta, T.; Galán, T.; et al. Broadband image sensor array based on graphene-CMOS integration. Nat. Photon. 2017, 11, 366-371. [CrossRef]

28. Wu, K.; Park, Y.; Lim, J.; Klimov, V.I. Towards zero-threshold optical gain using charged semiconductor quantum dots. Nat. Nanotechnol. 2017, 12, 1140-1147. [CrossRef]

29. Yuan, F.; Yuan, T.; Sui, L.; Wang, Z.; Xi, Z.; Li, Y.; Li, X.; Fan, L.; Tan, Z.; Chen, A.; et al. Engineering triangular carbon quantum dots with unprecedented narrow bandwidth emission for multicolored LEDs. Nat. Commun. 2018, 9, 2249. [CrossRef]

30. Böberl, M.; Kovalenko, M.V.; Gamerith, S.; List, E.J.W.; Heiss, W. Inkjet-printed nanocrystal photodetectors operating up to $3 \mu \mathrm{m}$ wavelengths. Adv. Mater. 2007, 19, 3574-3578. [CrossRef]

31. Chen, M.; Lu, H.; Abdelazim, N.M.; Zhu, Y.; Wang, Z.; Ren, W.; Kershaw, S.V.; Rogach, A.L.; Zhao, N. Mercury telluride quantum dot based phototransistor enabling high-sensitivity room-temperature photodetection at $2000 \mathrm{~nm}$. ACS Nano 2017, 11, 5614-5622. [CrossRef] [PubMed]

32. Jagtap, A.; Goubet, N.; Livache, C.; Chu, A.; Martinez, B.; Gréboval, C.; Qu, J.; Dandeu, E.; Becerra, L.; Witkowski, N.; et al. Short wave infrared devices based on HgTe nanocrystals with air stable performances. J. Phys. Chem. C 2018, 122, 14979-14985. [CrossRef]

33. Ackerman, M.M.; Chen, M.; Guyot-Sionnest, P. HgTe colloidal quantum dot photodiodes for extended short-wave infrared detection. Appl. Phys. Lett. 2020, 116, 083502. [CrossRef]

34. Keuleyan, S.; Lhuillier, E.; Brajuskovic, V.; Guyot-Sionnest, P. Mid-infrared HgTe colloidal quantum dot photodetectors. Nat. Photonics 2011, 5, 489-493. [CrossRef]

35. Keuleyan, S.; Lhuillier, E.; Guyot-Sionnest, P. Synthesis of colloidal HgTe quantum dots for narrow mid-IR emission and detection. J. Am. Chem. Soc. 2011, 133, 16422-16424. [CrossRef] [PubMed]

36. Lhuillier, E.; Keuleyan, S.; Guyot-Sionnest, P. Colloidal quantum dots for mid-IR applications. Infrared Phys. Technol. 2013, 59, 133-136. [CrossRef]

37. Lhuillier, E.; Keuleyan, S.; Zolotavin, P.; Guyot-Sionnest, P. Mid-infrared HgTe/ $\mathrm{As}_{2} \mathrm{~S}_{3}$ field effect transistors and photodetectors. Adv. Mater. 2013, 25, 137-141. [CrossRef]

38. Guyot-Sionnest, P.; Roberts, J.A. Background limited mid-infrared photodetection with photovoltaic HgTe colloidal quantum dots. Appl. Phys. Lett. 2015, 107, 253104. [CrossRef]

39. Yifat, Y.; Ackerman, M.; Guyot-Sionnest, P. Mid-IR colloidal quantum dot detectors enhanced by optical nano-antennas. Appl. Phys. Lett. 2017, 110, 41106. [CrossRef]

40. Ackerman, M.M.; Tang, X.; Guyot-Sionnest, P. Fast and sensitive colloidal quantum dot mid-wave infrared photodetectors. ACS Nano 2018, 12, 7264-7271. [CrossRef]

41. Tang, X.; Ackerman, M.M.; Guyot-Sionnest, P. Thermal Imaging with Plasmon Resonance Enhanced HgTe Colloidal Quantum Dot Photovoltaic Devices. ACS Nano 2018, 12, 7362-7370. [CrossRef] [PubMed]

42. Chen, M.; Lan, X.; Tang, X.; Wang, Y.; Hudson, M.H.; Talapin, D.V.; Guyot-Sionnest, P. High carrier mobility in HgTe quantum dot solids improves mid-IR photodetectors. ACS Photonics. 2019, 6, 2358-2365. [CrossRef]

43. Keuleyan, S.E.; Guyot-Sionnest, P.; Delerue, C.; Allan, G. Mercury telluride colloidal quantum dots: Electronic structure, size-dependent spectra, and photocurrent detection up to $12 \mu \mathrm{m}$. ACS Nano 2014, 8 , 8676-8682. [CrossRef] [PubMed]

44. Tang, X.; Wu, G.F.; Lai, K.W.C. Plasmon resonance enhanced colloidal HgSe quantum dot filterless narrowband photodetectors for mid-wave infrared. J. Mater. Chem. C 2017, 5, 362-369. [CrossRef]

45. Goubet, N.; Jagtap, A.; Livache, C.; Martinez, B.; Portalès, H.; Xu, X.Z.; Lobo, R.P.S.M.; Dubertret, B.; Lhuillier, E. Terahertz HgTe nanocrystals: Beyond confinement. J. Am. Chem. Soc. 2018, 140, 5033-5036. [CrossRef]

46. Lhuillier, E.; Scarafagio, M.; Hease, P.; Nadal, B.; Aubin, H.; Xu, X.Z.; Lequeux, N.; Patriarche, G.; Ithurria, S.; Dubertret, B. Infrared photodetection based on colloidal quantum-dot films with high mobility and optical absorption up to THz. Nano Lett. 2016, 16, 1282-1286. [CrossRef]

47. Lhuillier, E.; Keuleyan, S.; Guyot-Sionnest, P. Optical properties of HgTe colloidal quantum dots. Nanotechnology 2012, 23, 175705. [CrossRef]

48. Shen, G.; Chen, M.; Guyot-Sionnest, P. Synthesis of nonaggregating HgTe colloidal quantum dots and the emergence of air-stable n-doping. J. Phys. Chem. Lett. 2017, 8, 2224-2228. [CrossRef]

49. Tang, X.; Tang, X.; Lai, K.W.C. Scalable fabrication of infrared detectors with multispectral photoresponse based on patterned colloidal quantum dot films. ACS Photonics 2016, 3, 2396-2404. [CrossRef] 
50. Liu, H.; Lhuillier, E.; Guyot-Sionnest, P. 1/f noise in semiconductor and metal nanocrystal solids. J. Appl. Phys. 2014, 115, 154309. [CrossRef]

51. Nikitskiy, I.; Goossens, S.; Kufer, D.; Lasanta, T.; Navickaite, G.; Koppens, F.H.L.; Konstantatos, G. Integrating an electrically active colloidal quantum dot photodiode with a graphene phototransistor. Nat. Commun. 2016, 7, 11954. [CrossRef] [PubMed]

52. Huo, N.; Gupta, S.; Konstantatos, G. $\mathrm{MoS}_{2}-\mathrm{HgTe}$ Quantum dot hybrid photodetectors beyond $2 \mu \mathrm{m}$. Adv. Mater. 2017, 29, 1606576. [CrossRef] [PubMed]

53. Özdemir, O.; Ramiro, I.; Gupta, S.; Konstantatos, G. High sensitivity hybrid PbS CQD-TMDC photodetectors up to $2 \mu \mathrm{m}$. ACS Photonics 2019, 6, 2381-2386. [CrossRef]

54. Xu, H.; Wu, J.; Feng, Q.; Mao, N.; Wang, C.; Zhang, J. High responsivity and gate tunable graphene-MoS 2 hybrid phototransistor. Small 2014, 10, 2300-2306. [CrossRef] [PubMed]

55. Chen, M.; Shao, L.; Kershaw, S.V.; Yu, H.; Wang, J.; Rogach, A.L.; Zhao, N. Photocurrent enhancement of HgTe quantum dot photodiodes by plasmonic gold nanorod structures. ACS Nano 2014, 8, 8208-8216. [CrossRef] [PubMed]

56. Tang, X.; Ackerman, M.M.; Shen, G.; Guyot-Sionnest, P. Towards infrared electronic eyes: Flexible colloidal quantum dot photovoltaic detectors enhanced by resonant cavity. Small 2019, 15, 1804920. [CrossRef]

57. Tang, X.; Ackerman, M.M.; Guyot-Sionnest, P. Acquisition of hyperspectral data with colloidal quantum dots. Laser Photonics Rev. 2019, 13, 1900165. [CrossRef]

58. Tang, X.; Ackerman, M.M.; Chen, M.; Guyot-Sionnest, P. Dual-band infrared imaging using stacked colloidal quantum dot photodiodes. Nat. Photonics 2019, 13, 277-282. [CrossRef]

59. Choi, J.; Wang, H.; Oh, S.J.; Paik, T.; Jo, P.S.; Sung, J.; Ye, X.; Zhao, T.; Diroll, B.T.; Murray, C.B.; et al. Exploiting the colloidal nanocrystal library to construct electronic devices. Science 2016, 352, 205-208. [CrossRef]

60. Tang, X.; Chen, M.; Kamath, A.; Ackerman, M.M.; Guyot-Sionnest, P. Colloidal quantum-dots/graphene/silicon dual-channel detection of visible light and short-wave infrared. ACS Photonics 2020, 7, 1117-1121. [CrossRef]

61. Kim, L.; Anikeeva, P.O.; Coe-Sullivan, S.A.; Steckel, J.S.; Bawendi, M.G.; Bulovic, V. Contact printing of quantum dot light-emitting devices. Nano Lett. 2008, 8, 4513-4517. [CrossRef]

62. Kim, T.; Cho, K.; Lee, E.K.; Lee, S.J.; Chae, J.; Kim, J.W.; Kim, D.H.; Kwon, J.; Amaratunga, G.; Lee, S.Y.; et al. Full-colour quantum dot displays fabricated by transfer printing. Nat. Photonics 2011, 5, 176-182. [CrossRef]

63. Choi, M.K.; Yang, J.; Kang, K.; Kim, D.C.; Choi, C.; Park, C.; Kim, S.J.; Chae, S.I.; Kim, T.; Kim, J.H.; et al. Wearable red-green-blue quantum dot light-emitting diode array using high- resolution intaglio transfer printing. Nat. Commun. 2015, 6, 7149. [CrossRef] [PubMed]

64. Haverinen, H.M.; Myllylä, R.A.; Jabbour, G.E. Inkjet printing of light emitting quantum dots. Appl. Phys. Lett. 2009, 94, 73108. [CrossRef]

65. Wood, V.; Panzer, M.J.; Chen, J.; Bradley, M.S.; Halpert, J.E.; Bawendi, M.G.; Bulovic, V. Inkjet-printed quantum dot-polymer composites for full-color AC-driven displays. Adv. Mater. 2009, 21, 2151-2155. [CrossRef]

66. Zhang, H.; Son, J.S.; Dolzhnikov, D.S.; Filatov, A.S.; Hazarika, A.; Wang, Y.; Hudson, M.H.; Sun, C.; Chattopadhyay, S.; Talapin, D.V. Soluble lead and bismuth chalcogenidometallates: Versatile solders for thermoelectric materials. Chem. Mater. 2017, 29, 6396-6404. [CrossRef]

67. Bertino, M.F.; Gadipalli, R.R.; Story, J.G.; Williams, C.G.; Zhang, G.; Sotiriou-Leventis, C.; Tokuhiro, A.T.; Guha, S.; Leventis, N. Laser writing of semiconductor nanoparticles and quantum dots. Appl. Phys. Lett. 2004, 85, 6007-6009. [CrossRef]

68. Wang, Y.; Fedin, I.; Zhang, H.; Talapin, D.V. Direct optical lithography of functional inorganic nanomaterials. Science 2017, 357, 385-388. [CrossRef]

69. Wang, Y.; Pan, J.; Wu, H.; Talapin, D.V. Direct wavelength-selective optical and electron-beam lithography of functional inorganic nanomaterials. ACS Nano 2019, 13, 13917-13931. [CrossRef]

70. Kim, J.; Kwon, S.; Kang, Y.K.; Kim, Y.; Lee, M.; Han, K.; Facchetti, A.; Kim, M.; Park, S.K. A skin-like two-dimensionally pixelized full-color quantum dot photodetector. Sci. Adv. 2019, 5, eaax8801. [CrossRef]

71. Tang, X.; Chen, M.; Ackerman, M.M.; Melnychuk, C.; Guyot-Sionnest, P. Direct imprinting of quasi-3D nanophotonic structures into colloidal quantum-dot devices. Adv. Mater. 2020, 32, 1906590. [CrossRef] [PubMed] 
72. Ciani, A.J.; Pimpinella, R.E.; Grein, C.H.; Guyot-Sionnest, P. Colloidal quantum dots for low-cost MWIR imaging. In Infrared Technology and Applications XLII, Proceedings of SPIE Defense + Security, Baltimore, MD, USA, 20 May 2016; SPIE: Bellingham, WA, USA, 2016; Volume 9819, p. 981919. [CrossRef]

73. Buurma, C.; Pimpinella, R.E.; Ciani, A.J.; Feldman, J.S.; Grein, C.H.; Guyot-Sionnest, P. MWIR imaging with low cost colloidal quantum dot films. In Optical Sensing, Imaging, and Photon Counting: Nanostructured Devices and Applications 2016, Proceedings of SPIE Nanoscience + Engineering, San Diego, CA, USA,26 September 2016; SPIE: Bellingham, WA, USA, 2016; Volume 9933, p. 993303. [CrossRef]

(C) 2020 by the authors. Licensee MDPI, Basel, Switzerland. This article is an open access article distributed under the terms and conditions of the Creative Commons Attribution (CC BY) license (http://creativecommons.org/licenses/by/4.0/). 